\title{
Hard Squares with Negative Activity on Cylinders with Odd Circumference
}

\author{
Jakob Jonsson* \\ Department of Mathematics \\ KTH, Stockholm, Sweden \\ jakobj@kth.se \\ Submitted: Sep 30, 2008; Accepted: Mar 13, 2009; Published: Mar 23, 2009 \\ Mathematics Subject Classification: 05A15, 05C69, 52C20
}

Dedicated to Anders Björner on the occasion of his 60th birthday.

\begin{abstract}
Let $C_{m, n}$ be the graph on the vertex set $\{1, \ldots, m\} \times\{0, \ldots, n-1\}$ in which there is an edge between $(a, b)$ and $(c, d)$ if and only if either $(a, b)=(c, d \pm 1)$ or $(a, b)=(c \pm 1, d)$, where the second index is computed modulo $n$. One may view $C_{m, n}$ as a unit square grid on a cylinder with circumference $n$ units. For odd $n$, we prove that the Euler characteristic of the simplicial complex $\Sigma_{m, n}$ of independent sets in $C_{m, n}$ is either 2 or -1 , depending on whether or not $\operatorname{gcd}(m-1, n)$ is divisble by 3 . The proof relies heavily on previous work due to Thapper, who reduced the problem of computing the Euler characteristic of $\Sigma_{m, n}$ to that of analyzing a certain subfamily of sets with attractive properties. The situation for even $n$ remains unclear. In the language of statistical mechanics, the reduced Euler characteristic of $\Sigma_{m, n}$ coincides with minus the partition function of the corresponding hard square model with activity -1 .
\end{abstract}

\section{Introduction}

An independent set in a simple and loopless graph $G$ is a subset of the vertex set of $G$ with the property that no two vertices in the subset are adjacent. The family of independent sets in $G$ forms a simplicial complex, the independence complex $\Sigma(G)$ of $G$.

The purpose of this paper is to analyze the independence complex of square grids with cylindrical boundary conditions. Specifically, define $C_{m}$ to be the graph with vertex set

${ }^{*}$ Research financed by the Swedish Research Council. Part of this research was carried out at the Erwin Schrödinger International Institute for Mathematical Physics in Vienna within the programme Combinatorics and Statistical Physics. 
$[m] \times \mathbb{Z}$ and with an edge between $(a, b)$ and $(c, d)$ if and only if either $(a, b)=(c, d \pm 1)$ or $(a, b)=(c \pm 1, d)$. Define $C_{m, n}$ by identifying the vertices $(a, b)$ and $(a, d)$ whenever $d-b$ is a multiple of $n$. Equivalently, the vertex set of $C_{m, n}$ is the set of cosets of $\{0\} \times n \mathbb{Z}$ in $[m] \times \mathbb{Z}$, meaning that each vertex is of the form $\{(i, j+k n): k \in \mathbb{Z}\}$ for some $i \in[m]$ and $j \in \mathbb{Z}$. We write

$$
\langle i, j\rangle=\{(i, j+k n): k \in \mathbb{Z}\} .
$$

There is an edge between two vertices $\langle a, b\rangle$ and $\langle c, d\rangle$ in $C_{m, n}$ if and only if there are integers $r$ and $s$ such that $(a, b+r n)$ and $(c, d+s n)$ form an edge in $C_{m}$.

To avoid misconceptions, we state already at this point that we label elements in $\mathbb{Z}^{2}$ according to the matrix convention; $(i, j)$ is the element in the $i$ th row below row 0 and the $j$ th column to the right of column 0 .

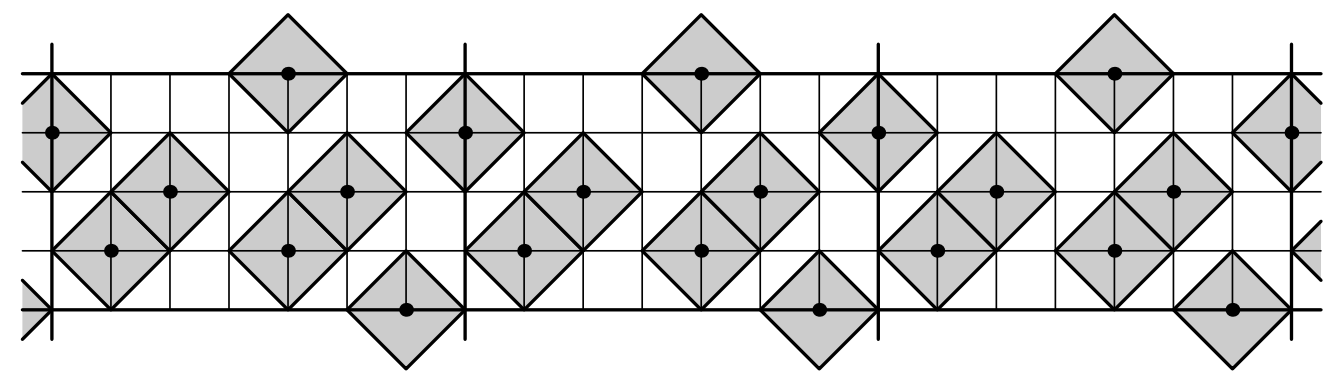

Figure 1: Configuration of hard squares invariant under translation with the vector $(0,7)$. The corresponding member of $\Sigma_{5,7}$ is the set of cosets of [5] $\times 7 \mathbb{Z}$ containing the square centers.

Properties of $\Sigma_{m, n}:=\Sigma\left(C_{m, n}\right)$ were discussed by Fendley, Schoutens, and van Eerten [6] in the context of the "hard square model" in statistical mechanics. This model deals with configurations of non-overlapping ("hard") squares in $\mathbb{R}^{2}$ such that the four corners of any square in the configuration coincide with the four neighbors $(x, y \pm 1)$ and $(x \pm 1, y)$ of a lattice point $(x, y) \in[m] \times \mathbb{Z}$. Identifying each such square with its center $(x, y)$, one obtains a bijection between members of the complex $\Sigma_{m, n}$ and hard square configurations that are invariant under the translation map $(x, y) \mapsto(x, y+n)$. See Figure 1 for an example.

Let $\Delta$ be a family of subsets of a finite set. Borrowing terminology from statistical mechanics, we define the partition function $Z(\Delta ; z)$ of $\Delta$ as

$$
Z(\Delta ; z):=\sum_{\sigma \in \Delta} z^{|\sigma|} .
$$

Observe that the coefficient of $z^{k}$ in $Z(\Delta ; z)$ is the number of sets in $\Delta$ of size $k$. In particular, if $\Delta$ is a simplicial complex, then $-Z(\Delta ;-1)$ coincides with the reduced Euler characteristic of $\Delta$. Write $Z(\Delta):=Z(\Delta ;-1)$.

In a previous paper, the following was conjectured: 
Conjecture 1.1 (Jonsson [8]) For odd n, we have that

$$
Z\left(\Sigma_{m, n}\right)=\left\{\begin{array}{cl}
-2 & \text { if } 3 \text { divides } \operatorname{gcd}(m-1, n) \\
1 & \text { otherwise. }
\end{array}\right.
$$

Our goal is to prove this conjecture, following the approach of Thapper [11, §2.4]. Specifically, Thapper defined a matching on $\Sigma_{m, n}$, pairing odd sets (i.e., sets of odd size) with even sets, and reduced Conjecture 1.1 to a conjecture about $Z\left(Q_{2}\right)$ being zero for a certain subfamily $Q_{2}$ of $\Sigma_{m, n}$ whenever $n$ is odd. We obtain our proof by defining a matching on $Q_{2}$, and this matching is perfect whenever $n$ is odd.

Our approach does not seem to explain very well what is going on for even $n$. In particular, the important question whether $\left\{Z\left(\Sigma_{m, n}\right): n \geq 1\right\}$ forms a periodic sequence for each $m$ remains unanswered. Computational evidence for small $m$ [8] suggests that this sequence is indeed periodic.

In the case that 3 does not divide $\operatorname{gcd}(m-1, n)$, the conjecture is equivalent to saying that the unreduced Euler characteristic $\chi\left(\Sigma_{m, n}\right):=-Z\left(\Sigma_{m, n}\right)+1$ vanishes. In the paper just cited [8], it was shown that the same is true for a slightly different complex whenever $\operatorname{gcd}(m, n)=1$. The complex under consideration was a torical variant of $\Sigma_{m, n}$ obtained by adding edges between $(1, j)$ and $(m, j)$ for all $j$.

There has been quite some activity recently pertaining to the problem of computing the activity at -1 for various lattices, both among physicists $[1,5,6,3]$ and among combinatorialists $[2,4,8,9,11]$. For a very good overview of the physical background and further references, we refer to Huijse and Schoutens [7]. In the context of the present paper, the work of Bousquet-Mélou, Linusson and Nevo [2] is worth a particular mention. They consider a variant of $\Sigma_{m, n}$, roughly obtained via a 45 degree rotation, and obtain results not only about the Euler characteristic but also about the homotopy type and homology.

\section{Acknowledgments}

I thank Svante Linusson and Johan Thapper for interesting discussions and also for introducing me to the approach $[11, \S 2.4]$. I also thank an anonymous referee for an extremely careful review and several useful comments and remarks.

\section{Conventions for figures}

Before proceeding, we introduce some conventions for figures, which we will use throughout the remainder of the paper.

We identify each point in $[m] \times \mathbb{Z}_{n}$ or $[m] \times \mathbb{Z}$ with a unit square; two vertices being joined by an edge means that the corresponding squares share a common side. In any picture illustrating an independent set $\sigma$ restricted to a given piece of $[m] \times \mathbb{Z}_{n}$ or $[m] \times \mathbb{Z}$, the following conventions apply for a given vertex $x$ :

- $x \in \sigma$ : there is a large dark disk on the square representing $x$. 


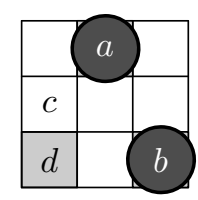

Figure 2: The restriction of a set $\sigma$ to a $3 \times 3$ piece of $[m] \times \mathbb{Z}_{n}$ or $[m] \times \mathbb{Z}$. The squares $a$ and $b$ marked with dark gray disks belong to $\sigma$, the white square $c$ does not belong to $\sigma$, and the status is unknown or unimportant for the gray square $d$. All other squares in the figure are neighbors of either $a$ or $b$ and do not belong to $\sigma$ if $\sigma$ is independent.

- $x \notin \sigma$ : the square is white.

- The status of $x$ is unknown or unimportant: the square is gray.

See Figure 2 for an example.

\section{The approach of Thapper}

We describe Thapper's approach [11] to proving Conjecture 1.1 and explain what remains to prove the conjecture. We also introduce some notation that we will use in later sections.

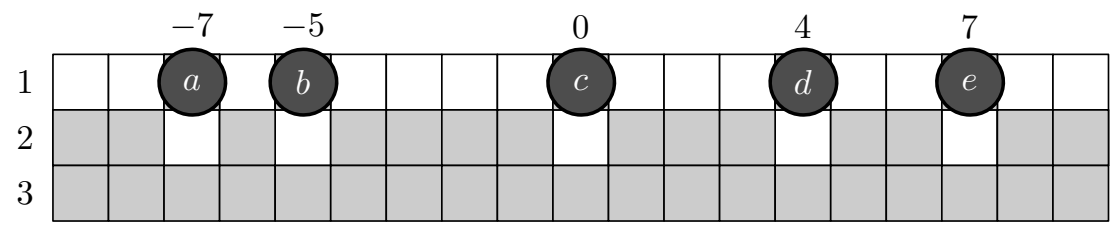

Figure 3: We have that $p_{\sigma}(7)=p_{\sigma}(6)=p_{\sigma}(5)=4$ and $p_{\sigma}(4)=p_{\sigma}(3)=p_{\sigma}(2)=p_{\sigma}(1)=0$. The vertices $b$ and $d$ are in even positions, whereas $c$ and $e$ are in odd positions.

For the time being, we make no assumptions about the parity of $n$, which hence may be any odd or even positive integer. For $\sigma \in \Sigma_{m, n}$, define $\pi(\sigma)$ to be the set of elements in $\sigma$ that appear in the first row;

$$
\pi(\sigma)=\sigma \cap\left\{\langle 1, j\rangle: j \in \mathbb{Z}_{n}\right\},
$$

where $\langle 1, j\rangle$ is defined as in $(1)$.

Assume that $\pi(\sigma)$ is nonempty. For each $j \in \mathbb{Z}$, let $p=p_{\sigma}(j)$ be maximal such that $p<j$ and $\langle 1, p\rangle \in \pi(\sigma)$. Clearly, $p_{\sigma}(j+n r)=p_{\sigma}(j)+n r$ for each integer $r$. Following Thapper [11], we refer to an element $\langle 1, j\rangle$ as being in an even position in $\sigma$ if $j-p_{\sigma}(j)$ is even; otherwise $\langle 1, j\rangle$ is in an odd position. Define $\pi_{o}(\sigma)$ to be the subset of $\pi(\sigma)$ consisting of those $\langle 1, j\rangle$ that are in an odd position and let $\pi_{e}(\sigma)=\pi(\sigma) \backslash \pi_{o}(\sigma)$. See Figure 3 for an illustration. 
An element $x$ is free with respect to $\sigma$ if no neighbor of $x$ is contained in $\sigma$. This means that we can add $x$ to $\sigma$ and stay in $\Sigma_{m, n}$. Otherwise, we say that $x$ is blocked in $\sigma$. By convention, all elements outside the cylinder $C_{m, n}$ are blocked. Define $\hat{\pi}_{e}(\sigma)$ to be the set of elements in even positions in the first row, inside or outside $\sigma$, that are free with respect to $\sigma$. If $\pi(\sigma)=\emptyset$, then we define $\hat{\pi}_{e}(\sigma)=\emptyset$. Again following Thapper, we define

$$
\hat{\sigma}=\sigma \cup \hat{\pi}_{e}(\sigma) .
$$

This means that $\pi(\hat{\sigma})=\pi_{o}(\sigma) \cup \hat{\pi}_{e}(\sigma)$. Let $X_{\sigma}$ be the family of sets $\tau$ such that $\hat{\sigma}=\hat{\tau}$.

Lemma 3.1 Suppose that $\pi_{o}(\sigma)$ is nonempty and assume that $x \in \hat{\pi}_{e}(\sigma)$. Then $\pi_{o}(\sigma \backslash$ $\{x\})=\pi_{o}(\sigma \cup\{x\})$ and $\hat{\pi}_{e}(\sigma \backslash\{x\})=\hat{\pi}_{e}(\sigma \cup\{x\})$. In particular, $\pi_{o}(\sigma)=\pi_{o}(\hat{\sigma})$ and $\hat{\pi}_{e}(\sigma)=\hat{\pi}_{e}(\hat{\sigma})$.

Proof. First, assume that we remove a vertex $\langle 1, j\rangle$ from $\pi_{e}(\sigma)$ to obtain a new set $\rho$. Then $\pi_{o}(\rho)=\pi_{o}(\sigma)$. Namely, let $\langle 1, k\rangle \in \pi(\rho)$ be such that $p_{\sigma}(k)=j$. We obtain that

$$
k-p_{\rho}(k)=k-p_{\sigma}(j)=(k-j)+\left(j-p_{\sigma}(j)\right) \equiv k-p_{\sigma}(k) \quad(\bmod 2),
$$

because $j-p_{\sigma}(j)$ is even by assumption. In particular, $\langle 1, k\rangle \in \pi_{o}(\rho)$ if and only if $\langle 1, k\rangle \in \pi_{o}(\sigma)$. Using the same argument, we deduce that $\hat{\pi}_{e}(\rho)=\hat{\pi}_{e}(\sigma)$; the neighbors of $\langle 1, j\rangle$ in the first row are in odd positions and thus remain outside $\hat{\pi}_{e}(\rho)$ even if they are free in $\rho$.

Next, assume that we form the set $\rho$ by adding a free vertex $\langle 1, j\rangle$ in an even position to $\sigma$. Then $\langle 1, j\rangle$ remains a free vertex in $\rho$. By the above discussion, we obtain the desired result.

Lemma 3.2 (Thapper [11, Lemma 4.1]) $\hat{\pi}_{e}(\sigma)$ is empty whenever $\pi_{o}(\sigma)$ is nonempty and $X_{\sigma}=\{\sigma\}$.

Proof. By Lemma 3.1, $\hat{\pi}_{e}(\sigma \backslash\{x\})=\hat{\pi}_{e}(\sigma \cup\{x\})$ for every $x \in \hat{\pi}_{e}(\sigma)$. In particular, $\sigma \backslash\{x\}, \sigma \cup\{x\} \in X_{\sigma}$ for every such $x$. Since $X_{\sigma}=\{\sigma\}$, we conclude that $\hat{\pi}_{e}(\sigma)=\emptyset$.

Thapper partitioned $\Sigma_{m, n}$ into a number of subfamilies. We tweak Thapper's partition slightly by moving the elements in his family $Q_{3}$ to our family $P_{2}$.

- $P_{1}$ is the family of sets $\sigma$ such that $\left|X_{\sigma}\right|>1$ and $\pi_{o}(\sigma) \neq \emptyset$.

- $P_{2}$ is the family of sets $\sigma$ such that $\pi_{o}(\sigma)=\emptyset$.

- $P_{3}$ is the family of sets $\sigma$ such that $X_{\sigma}=\{\sigma\}$ and $\pi_{o}(\sigma) \neq \emptyset$.

- $Q_{1}$ is the subfamily of $P_{3}$ consisting of all sets $\sigma$ such that $j-p_{\sigma}(j)=3$ for all $\langle 1, j\rangle \in \pi(\sigma)$.

- $Q_{2}$ is the subfamily of $P_{3}$ consisting of all sets $\sigma$ such that $j-p_{\sigma}(j) \geq 5$ for some $\langle 1, j\rangle \in \pi(\sigma)$. 
By Lemma 3.2, $P_{3}$ is indeed the disjoint union of $Q_{1}$ and $Q_{2}$; the difference $j-p_{\sigma}(j)$ is odd for all $\langle 1, j\rangle \in \pi(\sigma)$.

Thapper obtained a formula for $Z(X)$ for $X \in\left\{P_{1}, P_{2}, Q_{1}\right\}$.

Proposition 3.3 (Thapper [11]) The following hold for all $m, n \geq 1$.

(a) $Z\left(P_{1}\right)=0$.

(b) $Z\left(P_{2}\right)= \begin{cases}-Z\left(\Sigma_{m-1, n}\right)+2 \cdot(-1)^{m n / 4} & \text { if } m \text { and } n \text { are even; } \\ -Z\left(\Sigma_{m-1, n}\right) & \text { if } m \text { is odd and } n \text { is even; } \\ Z\left(\Sigma_{m-1, n}\right) & \text { if } n \text { is odd. }\end{cases}$

(c) $Z\left(Q_{1}\right)= \begin{cases}0 & \text { if } m \bmod 3=0 \text { or } 3 \not n \\ 3 \cdot(-1)^{n / 3} & \text { if } m \bmod 3=1 \text { and } 3 \mid n ; \\ 3 & \text { if } m \bmod 3=2 \text { and } 3 \mid n\end{cases}$

Since Thapper's thesis is not easily available, we present a proof of Proposition 3.3 in an appendix at the end of the paper.

Corollary 3.4 For $n$ odd, we have that

$$
Z\left(\Sigma_{m, n}\right)=Z\left(\Sigma_{m-1, n}\right)+Z\left(Q_{2}\right)+\left\{\begin{array}{cl}
0 & \text { if } m \bmod 3=0 \text { or } 3 \not n \\
-3 & \text { if } m \bmod 3=1 \text { and } 3 \mid n \\
3 & \text { if } m \bmod 3=2 \text { and } 3 \mid n
\end{array}\right.
$$

Thapper [11] conjectured that

$$
Z\left(Q_{2}\right)=0
$$

whenever $n$ is odd and applied Corollary 3.4 to prove that his conjecture implies Conjecture 1.1. Specifically, this follows immediately from induction on $m$ and the well-known fact [10, Prop. 5.2] that Conjecture 1.1 is true for $m=1$. We prove Conjecture 1.1 by demonstrating that Thapper's conjecture (2) is indeed true.

Theorem 3.5 For all $m$ and all odd $n$, we have that $Z\left(Q_{2}\right)=0$.

The proof of Theorem 3.5 ranges over several sections. In Section 4, we define a matching on $Q_{2}$ such that a set $\sigma$ belongs to the family $\Lambda$ of unmatched sets precisely when $x+(1,-1)$ is blocked for each $x \in \sigma$. In Section 5 , we analyze $\Lambda$ and develop the tools necessary to process this family further. In Section 6 , we define a matching on $\Lambda$ such that the remaining family $\Pi$ has certain attractive properties. Specifically, in Section 7 , we show that $\Pi$ is empty unless $n$ is even. The matchings have the property that even sets are paired with odd sets, leaving us with the conclusion that $Z\left(Q_{2}\right)$ is indeed zero whenever $n$ is odd. 


\section{Reducing $Q_{2}$ to the family $\Lambda$ of sets $\sigma$ with totally blocked $\operatorname{sw}(\sigma)$}

To start with, we assume that $n$ is arbitrary, making no assumption about the parity of $n$. For any element $x$ in $[m] \times \mathbb{Z}$ (or in $\left.[m] \times \mathbb{Z}_{n}\right)$, define $\mathbf{s}(x):=x+(1,0)$ (south), $\mathrm{e}(x):=x+(0,1)$ (east), $\mathrm{n}(x):=x+(-1,0)$ (north), and $\mathrm{w}(x):=x+(0,-1)$ (west); recall our matrix convention for indexing elements in $\mathbb{Z}^{2}$. In this section, we show that there is a matching on $Q_{2}$, pairing even sets with odd sets, such that the unmatched sets are those $\sigma$ with the property that $\operatorname{sw}(x)$ is blocked for each $x \in \sigma$. Recall that this means that either a neighbor of $\mathrm{sw}(x)$ belongs to $\sigma$ or $\operatorname{sw}(x)$ lies outside $C_{m, n}$. We denote by $\Lambda$ the family of such sets.

As Thapper [11, Lemma 4.1] observed, a set $\sigma \in \Sigma_{m, n}$ belongs to $Q_{1} \cup Q_{2}$ if and only if $j-p_{\sigma}(j)$ is odd for all $\langle 1, j\rangle \in \pi(\sigma)$ and all $\langle 1, k\rangle$ in even positions are blocked, meaning that either $\langle 1, k+1\rangle$ or $\langle 2, k\rangle$ belongs to $\sigma$.

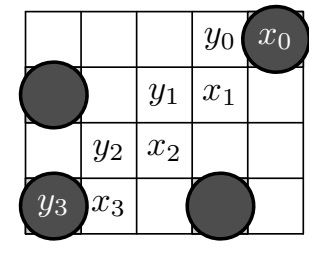

$\sigma$

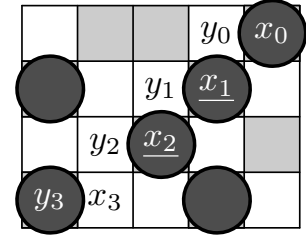

$(\mathrm{sw})^{*}(\sigma)$

Figure 4: $x_{1}$ and $x_{2}$ belong to $(\mathrm{sw})^{*}(\sigma)$; they are both free in $\sigma$, and $(\mathrm{sw})^{-2}\left(x_{2}\right)=$ $(\mathrm{sw})^{-1}\left(x_{1}\right)=x_{0} \in \sigma$. However, $x_{3}, y_{0}, y_{1}$, and $y_{2}$ do not belong to $(\mathrm{sw})^{*}(\sigma) ; x_{3}$ and $y_{0}$ are blocked, whereas $(\mathrm{sw})^{-2}\left(y_{2}\right)=(\mathrm{sw})^{-1}\left(y_{1}\right)=y_{0}$.

For a set $\sigma \in Q_{2}$, define $(\mathrm{sw})^{*}(\sigma)$ to be the set of elements $\langle a, b\rangle \in[m] \times \mathbb{Z}_{n}$ such that $(\mathrm{sw})^{-r}\langle a, b\rangle=(\mathrm{ne})^{r}\langle a, b\rangle=\langle a-r, b+r\rangle \in \sigma$ for some $r \in\{0, \ldots, a-1\}$ and such that

$$
(\mathrm{sw})^{-r}\langle a, b\rangle,(\mathrm{sw})^{-(r-1)}\langle a, b\rangle, \ldots,(\mathrm{sw})^{-1}\langle a, b\rangle,\langle a, b\rangle
$$

are all free in $\sigma$. See Figure 4 for an illustration. We say that $\langle a, b\rangle$ is $r$-free if this holds. Choose $r$ minimal with this property and define $\xi_{\sigma}\langle a, b\rangle=r$.

Lemma 4.1 We have that $(\mathrm{sw})^{*}(\sigma) \in Q_{2}$ whenever $\sigma \in Q_{2}$.

Proof. Assume the opposite; $(\mathrm{sw})^{*}(\sigma)$ contains two neighbors $x$ and $y$. By construction, $(\mathrm{sw})^{-r}(x)$ and $(\mathrm{sw})^{-s}(y)$ are free whenever $r \leq \xi_{\sigma}(x)$ and $s \leq \xi_{\sigma}(y)$. Now, $(\mathrm{sw})^{-\xi_{\sigma}(y)}(x)$ is blocked by $(\mathrm{sw})^{-\xi_{\sigma}(y)}(y)$ in $\sigma$ or in row 0 , which implies that $\xi_{\sigma}(x)<\xi_{\sigma}(y)$. However, we also have that $(\mathrm{sw})^{-\xi_{\sigma}(x)}(y)$ is blocked by $\left(\mathrm{sw}^{-\xi_{\sigma}(x)}(x)\right.$ in $\sigma$ or in row 0 , which implies that $\xi_{\sigma}(y)<\xi_{\sigma}(x)$, a contradiction.

Lemma 4.2 We have that $(\mathrm{sw})^{*}(\tau)=(\mathrm{sw})^{*}(\sigma)$ whenever $\sigma \subseteq \tau \subseteq(\mathrm{sw})^{*}(\sigma)$. 
Proof. It suffices to consider the case that $\tau=\sigma \cup\{y\}$ for some element $y$. First, assume that there is an element $z \in(\mathrm{sw})^{*}(\tau) \backslash(\mathrm{sw})^{*}(\sigma)$. The only possibility is that $z$ is $r$-free in $\tau$, where $r$ satisfies $(\mathrm{sw})^{-r}(z)=y$. However, since $y \in(\mathrm{sw})^{*}(\sigma)$, we have that $y$ is $s$-free in $\sigma$ for some $s \geq 1$. Since any free element in $\tau$ is also free in $\sigma$, it follows that $z$ is $(r+s)$-free in $\sigma$, meaning that $z \in(\mathrm{sw})^{*}(\sigma)$, a contradiction. Next, assume that there is an element $z \in(\mathrm{sw})^{*}(\sigma) \backslash(\mathrm{sw})^{*}(\tau)$. Then there is some $r$ such that $z$ is $r$-free in $\sigma$ but not in $\tau$. The only possibility is that $y$ blocks some element $(\mathrm{sw})^{-k}(z)$ for some $k \in\{0, \ldots, r\}$. However, $(\mathrm{sw})^{-k}(z)$ is $(r-k)$-free in $\sigma$. Since $(\mathrm{sw})^{*}(\sigma) \in \Sigma_{m, n}$ by Lemma 4.1, it follows that $(\mathrm{sw})^{-k}(z)$ is free in $\tau$, a contradiction.

Note that Lemma 4.2 implies that $(\mathrm{sw})^{*}\left((\mathrm{sw})^{*}(\sigma)\right)=(\mathrm{sw})^{*}(\sigma)$ for all $\sigma \in Q_{2}$. This means that $(\mathrm{sw})^{*}$ defines a closure operator on $Q_{2}$, viewed as a partially ordered set ordered by inclusion.

For any $\tau$ such that $(\mathrm{sw})^{*}(\tau)=\tau$, we define

$$
Q_{2}(\tau)=\left\{\sigma \in Q_{2}:(\mathrm{sw})^{*}(\sigma)=\tau\right\} .
$$

Assume that $x, y \in \tau$ and $y=\operatorname{sw}(x)$. Then $\sigma \backslash\{y\} \in Q_{2}(\tau)$ if and only if $\sigma \cup\{y\} \in Q_{2}(\tau)$. Namely, if $(\mathrm{sw})^{*}(\sigma \backslash\{y\})=\tau$, then Lemma 4.2 yields that $(\mathrm{sw})^{*}(\sigma)=(\mathrm{sw})^{*}(\sigma \backslash\{y\})=\tau$, because $y \in \tau$. Conversely, suppose that $(\mathrm{sw})^{*}(\sigma \cup\{y\})=\tau$. We have that $y \in(\mathrm{sw})^{*}(\sigma \backslash$ $\{y\})$. Namely, $x$ is $r$-free in $\sigma \cup\{y\}$ and hence also in $\sigma \backslash\{y\}$ for some $r$, because $y$ is equal to $\mathrm{sw}(x)$ and hence distinct from $(\mathrm{sw})^{-r}(x)$ for all nonnegative $r$. It follows that $y$ is $(r+1)$-free in $\sigma \backslash\{y\}$. Again, Lemma 4.2 yields that $(\mathrm{sw})^{*}(\sigma \backslash\{y\})=(\mathrm{sw})^{*}(\sigma \cup\{y\})=\tau$.

Let $\Lambda$ be the subfamily of $Q_{2}$ consisting of those $\tau$ such that for each $x \in \tau$ we have that $\operatorname{sw}(x)$ is blocked in $\tau$. By Lemma 4.2, $\tau$ belongs to $\Lambda$ if and only if $(\mathrm{sw})^{*}(\tau)=\tau$ and $Q_{2}(\tau)=\{\tau\}$.

We conclude the following.

Lemma 4.3 Suppose that $\tau=(\mathrm{sw})^{*}(\tau)$ and that $\tau$ contains elements $x, y$ such that $y=$ $\mathrm{sw}(x)$. Then $Z\left(Q_{2}(\tau)\right)=0$. In particular,

$$
Z\left(Q_{2}\right)=Z(\Lambda)
$$

\section{$5 \quad$ Analyzing the family $\Lambda$}

Let $\tau \in \Lambda$. Recall that if $\langle 1, j\rangle,\langle 1, j+2 t+1\rangle \in \pi(\tau)$ and $p_{\sigma}(j+2 t+1)=j$, then $\langle 1, j+2 k\rangle$ is blocked in $\tau$ by some element not in the first row for $1 \leq k \leq t-1$. The only possible element is $\langle 2, j+2 k\rangle$. Let $\psi(\tau)$ be the set consisting of all $(2, i)$ such that $\langle 2, i\rangle$ is such a blocking element in $\tau$ and also all $(1, i)$ such that $\langle 1, i\rangle \in \tau$. Note that $\psi(\tau)$ is a subset of $\{1,2\} \times \mathbb{Z}$ rather than $\{1,2\} \times \mathbb{Z}_{n}$. We make this choice to facilitate analysis.

List the elements of $\psi(\tau)$ in increasing column order as

$$
\psi(\tau)=\left\{x_{i}:=\left(a_{i}, b_{i}\right):-\infty<i<\infty\right\} .
$$

Hence $b_{i}<b_{j}$ whenever $i<j$. Note that $a_{i} \in\{1,2\}$ for all $i$. See Figure 5 for an example. 


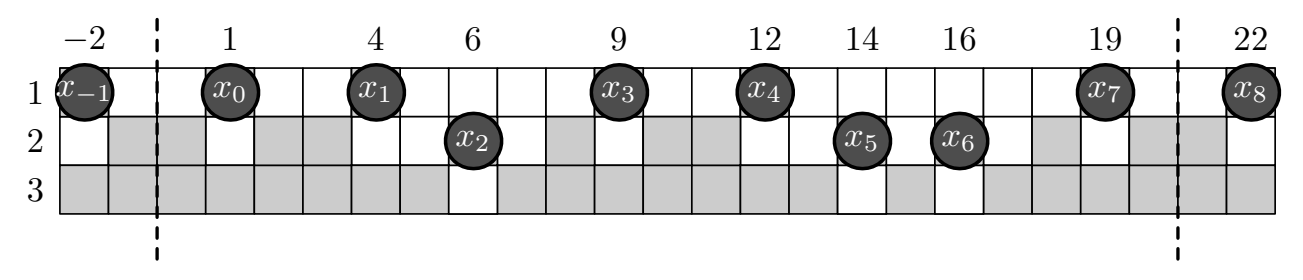

Figure 5: Illustrating example with $n=21$ and $m \geq 3$. We have that $\psi(\tau)=$ $\{(1,1),(1,4),(2,6),(1,9),(1,12),(2,14),(2,16),(1,19)\}+\{0\} \times 21 \mathbb{Z}$. Note that $\psi(\tau)$ does not contain any other elements $(2, i)$ such that $\langle 2, i\rangle \in \tau$.

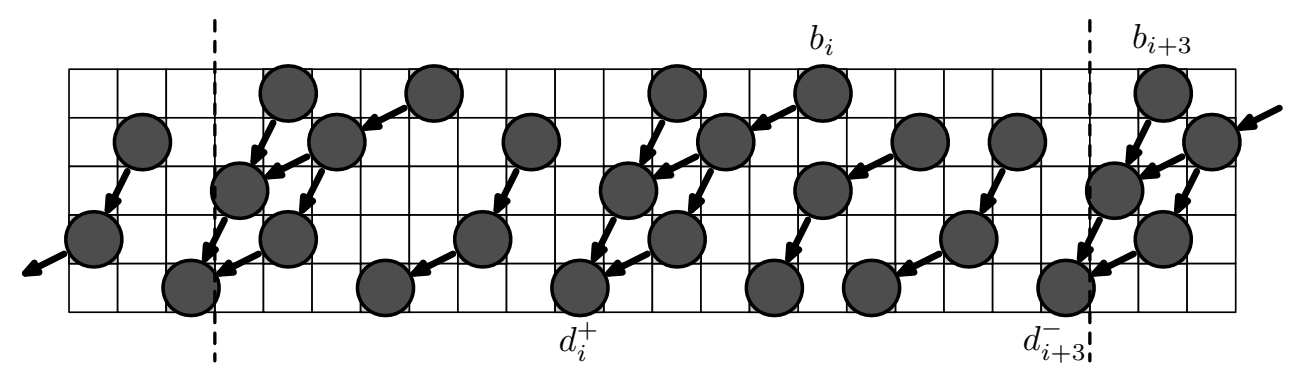

Figure 6: The digraph $D\left(\tau^{*}\right)$ for a certain $\tau \in \Lambda$ in the case that $m=5$ and $n=18$. Note that $d_{i+3}^{-}-d_{i}^{+}=b_{i+3}-b_{i}+3$ as predicted by Lemma 5.2 .

By construction, $\operatorname{sw}(y)$ is blocked in $\tau$ for every $y \in \tau$. In particular, unless $y$ lies in row $m$, either $\mathrm{s}^{2} \mathrm{w}(y)$ or $\mathbf{s w}^{2}(y)$ belongs to $\tau$. Let $\tau^{*}$ be the set of vertices $(r, s)$ in $[m] \times \mathbb{Z}$ such that $\langle r, s\rangle \in \tau$. Form a directed graph $D\left(\tau^{*}\right)$ on the vertex set $\tau^{*}$ by introducing an edge from a vertex $y$ to another vertex $z$ in $\tau^{*}$ whenever $z=\mathbf{s}^{2} \mathrm{w}(y)$ or $z=\mathbf{s w}^{2}(y)$. See Figure 6 for an example. It is an easy task to check that there is a directed path from $y$ to some element on row $m$ for each $y \in \tau^{*}$. Namely, if such a path stopped at row $k<m$ with an element $z$, then $\operatorname{sw}(z)$ would be free in $\tau$, a contradiction.

For each $i$, let $d_{i}^{-}$be minimal and let $d_{i}^{+}$be maximal such that there are paths from $x_{i}$ to $y_{i}^{-}:=\left(m, d_{i}^{-}\right)$and $y_{i}^{+}:=\left(m, d_{i}^{+}\right)$in $D\left(\tau^{*}\right)$. Note that $d_{i}^{-}$and $d_{i}^{+}$may well coincide.

For a vertex $y=(r, s)$, define $\nu(y):=s-r$. Now, we make the following key observation:

- If there is a path from $y=(a, b)$ to $z=(c, d)$, then $\nu(y)=b-a$ and $\nu(z)=d-c$ are congruent modulo three.

In particular, $\nu\left(x_{i}\right) \equiv \nu\left(y_{i}^{+}\right) \equiv \nu\left(y_{i}^{-}\right)(\bmod 3)$. Moreover, if $\nu\left(x_{i}\right)$ and $\nu\left(x_{j}\right)$ belong to different congruence classes modulo three, then a directed path starting in $x_{i}$ cannot intersect a directed path starting in $x_{j}$. Thus we have the following fact:

Lemma 5.1 If $\nu\left(x_{i+1}\right)-\nu\left(x_{i}\right) \equiv 1(\bmod 3)$, then

$$
\nu\left(y_{i+1}^{-}\right)-\nu\left(y_{i}^{+}\right)=d_{i+1}^{-}-d_{i}^{+} \geq 4 .
$$


If instead $\nu\left(x_{i+1}\right)-\nu\left(x_{i}\right) \equiv 2(\bmod 3)$, then

$$
\nu\left(y_{i+1}^{-}\right)-\nu\left(y_{i}^{+}\right)=d_{i+1}^{-}-d_{i}^{+} \geq 2 .
$$

For the former inequality, use the fact that $d_{i+1}^{-}-d_{i}^{+}$cannot be equal to 1 , as this would imply that $y_{i+1}^{-}$and $y_{i}^{+}$were neighbors.

Now, suppose that $x_{i}=(1, j), x_{i+k}=(2, j+2 k)$ for $1 \leq k \leq t-1$, and $x_{i+t}=$ $(1, j+2 t+1)$, where $2 t+1 \geq 5$. This means that $p_{\tau}\left(b_{i+t}\right)=b_{i}=b_{i+t}-(2 t+1)$. Note that

$$
\begin{aligned}
\nu\left(x_{i+1}\right)-\nu\left(x_{i}\right) & =1 ; \\
\nu\left(x_{i+k}\right)-\nu\left(x_{i+k-1}\right) & =2 \text { for } 2 \leq k \leq t-1 ; \\
\nu\left(x_{i+t}\right)-\nu\left(x_{i+t-1}\right) & =4 .
\end{aligned}
$$

By Lemma 5.1, we may conclude that

$$
\begin{aligned}
d_{i+1}^{-}-d_{i}^{+} & \geq \nu\left(x_{i+1}\right)-\nu\left(x_{i}\right)+3 \\
d_{i+k}^{-}-d_{i+k-1}^{+} & \geq \nu\left(x_{i+k}\right)-\nu\left(x_{i+k-1}\right) \text { for } 2 \leq k \leq t .
\end{aligned}
$$

Summing and using the trivial inequality $d_{i+k}^{+} \geq d_{i+k}^{-}$, we obtain the following lemma; see Figure 6 for an illustration.

Lemma 5.2 With notation as above, we have that

$$
d_{i+t}^{-}-d_{i}^{+} \geq \nu\left(x_{i+t}\right)-\nu\left(x_{i}\right)+3=2 t+4 .
$$

Consider an arbitrary index $i$. We have four possibilities for $x_{i}$ and $x_{i+1}$.

- $a_{i}=1, a_{i+1}=2$ and $b_{i+1}-b_{i}=2$, meaning that $\nu\left(x_{i+1}\right)-\nu\left(x_{i}\right)=1$.

- $a_{i}=a_{i+1}=2$ and $b_{i+1}-b_{i}=2$, meaning that $\nu\left(x_{i+1}\right)-\nu\left(x_{i}\right)=2$.

- $a_{i}=2, a_{i+1}=1$ and $b_{i+1}-b_{i}=3$, meaning that $\nu\left(x_{i+1}\right)-\nu\left(x_{i}\right)=4$.

- $a_{i}=a_{i+1}=1$ and $b_{i+1}-b_{i}=3$, meaning that $\nu\left(x_{i+1}\right)-\nu\left(x_{i}\right)=3$.

The last case is the only situation where $\nu\left(x_{i}\right)$ and $\nu\left(x_{i+1}\right)$ belong to the same congruence class modulo three. Write $x_{i} \sim x_{i+1}$ if this is the case and extend $\sim$ to an equivalence relation. If $x_{i-1} \nsim x_{i} \sim x_{i+t} \nsim x_{i+t+1}$, then we refer to $\left\{x_{i}, \ldots, x_{i+t}\right\}$ as a block. $x_{i}$ and $x_{i+t}$ are the boundary points of the block, whereas $x_{i+1}, \ldots, x_{i+t-1}$ are the interior points. In a singleton block $\left\{x_{i}\right\}$, both boundary points coincide with the one element $x_{i}$ in the block.

To better understand the structure of $D\left(\tau^{*}\right)$, we prove a result about its connected components.

Lemma 5.3 Suppose that $i<j$ and $x_{i} \not x_{j}$. Then, $x_{i}$ and $x_{j}$ belong to different connected components in $D\left(\tau^{*}\right)$. If , in addition, $\nu\left(x_{i}\right) \equiv \nu\left(x_{j}\right)(\bmod 3)$, then there is a third connected component, containing some $x_{k}$ such that $i<k<j$, that separates the two components containing $x_{i}$ and $x_{j}$. 
Proof. The statement is obvious in the case that $\nu\left(x_{i}\right) \not \equiv \nu\left(x_{j}\right)(\bmod 3)$, because $\nu(y) \bmod 3$ is the same for all elements $y$ in a given component. Moreover, if indeed $\nu\left(x_{i}\right) \equiv \nu\left(x_{j}\right)(\bmod 3)$, then there must be an index $k$ such that $i<k<j$ and such that $\nu\left(x_{i}\right) \not \equiv \nu\left(x_{k}\right)$, because otherwise $x_{i} \sim x_{j}$. It is clear that any path from $x_{k}$ to $y_{k}^{ \pm}$ separates the components containing $x_{i}$ and $x_{j}$; hence we are done.

Now, let $\psi_{0}(\tau)$ be the subset of $\psi(\tau)$ obtained by removing all elements in the second row and all interior points of blocks in the first row. The latter means that we remove all elements $x_{i}$ such that $x_{i-1}=\mathrm{w}^{3}\left(x_{i}\right)$ and $x_{i+1}=\mathrm{e}^{3}\left(x_{i}\right)$. List the elements of $\psi_{0}(\tau)$ as

$$
\psi_{0}(\tau)=\left\{x_{i_{r}}=\left(1, b_{i_{r}}\right):-\infty<r<\infty\right\}
$$

in increasing column order. For each $r$, we have exactly one of the following two situations:

- $b_{i_{r}}-b_{i_{r-1}}$ is equal to $2\left(i_{r}-i_{r-1}\right)+1 \geq 5$ and $x_{i_{r-1}+1}, \ldots, x_{i_{r}-1}$ all lie in the second row with one vertex in every other column between $b_{i_{r-1}}+2$ and $b_{i_{r}}-3$. Let $\psi_{0}^{\geq 5}(\tau)$ be the set of such $x_{i_{r}}$.

- $b_{i_{r}}-b_{i_{r-1}}$ is equal to $3\left(i_{r}-i_{r-1}\right)$ and $x_{i_{r-1}+1}, \ldots, x_{i_{r}-1}$ all lie in the first row with one vertex in every third column between $b_{i_{r-1}}+3$ and $b_{i_{r}}-3$. Let $\psi_{0}^{3}(\tau)$ be the set of such $x_{i_{r}}$.

By Lemma 5.2,

$$
d_{i_{r}}^{-}-d_{i_{r-1}}^{+} \geq \nu\left(x_{i_{r}}\right)-\nu\left(x_{i_{r-1}}\right)+3
$$

whenever $x_{i_{r}} \in \psi_{0}^{\geq 5}(\tau)$. We want to define a matching on $\Lambda$ such that all remaining unmatched sets satisfy

$$
d_{i_{r}}^{+}-d_{i_{r-1}}^{-} \geq \nu\left(x_{i_{r}}\right)-\nu\left(x_{i_{r-1}}\right)-3 \epsilon_{r}
$$

whenever $x_{i_{r}} \in \psi_{0}^{3}(\tau)$, where $\epsilon_{r}=1$ if $i_{r}-i_{r-1}$ is odd and $\epsilon_{r}=0$ if $i_{r}-i_{r-1}$ is even. By the following lemma, this will imply the conjecture. We remark that Figure 6 provides an example of a set $\tau$ satisfying (3).

Lemma $5.4 A$ set $\tau \in \Lambda$ satisfying (3) must have the property that exactly every other $x_{i_{r}}$ belongs to $\psi_{0}^{\geq 5}(\tau)$. Moreover, for every $x_{i_{r}}$ in $\psi_{0}^{3}(\tau)$, we have that $b_{i_{r}}-b_{i_{r-1}}=3\left(i_{r}-i_{r-1}\right)$ is odd. In particular, $n$ is even.

Proof. Let $P$ be such that $x_{i_{r+P}}=\mathrm{e}^{n}\left(x_{i_{r}}\right)$ for all $r$; by periodicity of $\tau^{*}$, such a $P$ exists. Form a sum $S$ over $r$ with $r$ ranging from 1 to $P$ such that term number $r$ is $d_{i_{r}}^{-}-d_{i_{r-1}}^{+}$ if $x_{i_{r}} \in \psi_{0}^{\geq 5}(\tau)$ and $d_{i_{r}}^{+}-d_{i_{r-1}}^{-}$if $x_{i_{r}} \in \psi_{0}^{3}(\tau)$. Let $s_{r}$ and $t_{r-1}$ be signs such that term number $r$ is $d_{i_{r}}^{s_{r}}-d_{i_{r-1}}^{t_{r-1}}$.

We cannot have two consecutive elements $x_{i_{r}}$ and $x_{i_{r+1}}$ both appearing in $\psi_{0}^{3}(\tau)$, because then $x_{i_{r}-1}=\mathrm{w}^{3}\left(x_{i_{r}}\right)$ and $x_{i_{r}+1}=\mathrm{e}^{3}\left(x_{i_{r}}\right)$, which would mean that $x_{i_{r}} \notin \psi_{0}(\tau)$. By periodicity, the same is true for $x_{i_{P}}$ and $x_{1}$. 
As a consequence, $S \leq n$. Namely, $S$ is of the form

$$
\sum_{r=1}^{P}\left(d_{i_{r}}^{s_{r}}-d_{i_{r-1}}^{t_{r-1}}\right)=d_{i_{P}}^{s_{P}}-d_{i_{0}}^{t_{0}}+\sum_{r=1}^{P-1}\left(d_{i_{r}}^{s_{r}}-d_{i_{r}}^{t_{r}}\right)
$$

where $\left(s_{r}, t_{r}\right) \neq(+,-)$ for $1 \leq r \leq P-1$ and $\left(s_{P}, t_{0}\right) \neq(+,-)$; the latter is by periodicity.

Moreover, it also follows that the number $\alpha$ of elements in the set $\left\{x_{i_{r}}: 1 \leq r \leq P\right\}$ that belong to $\psi_{0}^{\geq 5}(\tau)$ is at least $\lceil P / 2\rceil$. Let $\beta$ be the number of elements $x_{i_{r}}$ in the same set that belong to $\psi_{0}^{3}(\tau)$ and have the property that $b_{i_{r}}-b_{i_{r-1}}$ is odd. Using Lemma 5.2 and (3), we deduce that

$$
\begin{aligned}
S & \geq \sum_{r=1}^{P}\left(b_{i_{r}}-b_{i_{r-1}}\right)+3 \alpha-3 \beta=b_{i_{P}}-b_{i_{0}}+3(\alpha-\beta) \\
& =n+3(\alpha-\beta) \geq S+3(\alpha-\beta) .
\end{aligned}
$$

Since $\alpha \geq P / 2 \geq \beta$, we must have that $\alpha=\beta=P / 2$. This implies that $P$ is even and $b_{i_{r}}-b_{i_{r-1}}$ is odd for $1 \leq r \leq P$. Since $n=\sum_{r=1}^{P}\left(b_{i_{r}}-b_{i_{r-1}}\right)$, we conclude that $n$ is even.

\section{Defining a matching on $\Lambda$}

Extend $\sim$ further by defining $y \sim z$ if there are directed paths from $x_{i}$ to $y$ and from $x_{j}$ to $z$ for some $x_{i}$ and $x_{j}$ such that $x_{i} \sim x_{j}$. Our matching on $\Lambda$ is defined in two steps.

\subsection{Step 1}

Let $\Lambda_{0}$ be the subfamily of $\Lambda$ consisting of all $\tau$ such that there are two vertices $u$ and $w:=(\mathbf{s e})^{2}(u)$ in $\tau^{*}$ such that $u \sim w$ and such that $\mathbf{s}^{2} \mathrm{w}(u), \mathbf{s w}^{2}(w) \notin \tau^{*}$. Write $\Gamma=\Lambda \backslash \Lambda_{0}$.

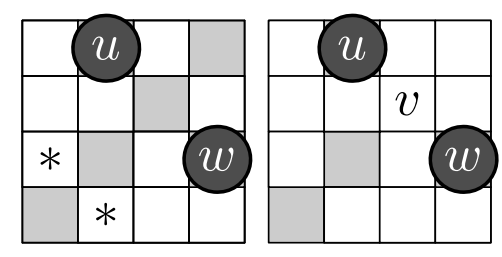

Figure 7: The situation in Step 1. The squares marked with stars in the left-hand figure are not present by assumption.

Lemma 6.1 We have that $Z\left(\Lambda_{0}\right)=0$. As a consequence, $Z(\Lambda)=Z(\Gamma)$. 
Proof. Among all pairs $(u, w)$ with properties as described above, choose the pair such that the row $a$ of $u=:(a, b)$ is minimal and, among the remaining pairs, such that the column $b$ is nonnegative and minimal.

See the picture on the left in Figure 7 for an illustration of the situation. By the assumption about every $y \in \tau^{*}$ having the property that $\operatorname{sw}(y)$ is blocked, we conclude that $\mathrm{e}^{2}(u)$ and $v:=\operatorname{se}(u)$ do not belong to $\tau^{*}$, which means that we have the situation in the picture on the right in the same figure.
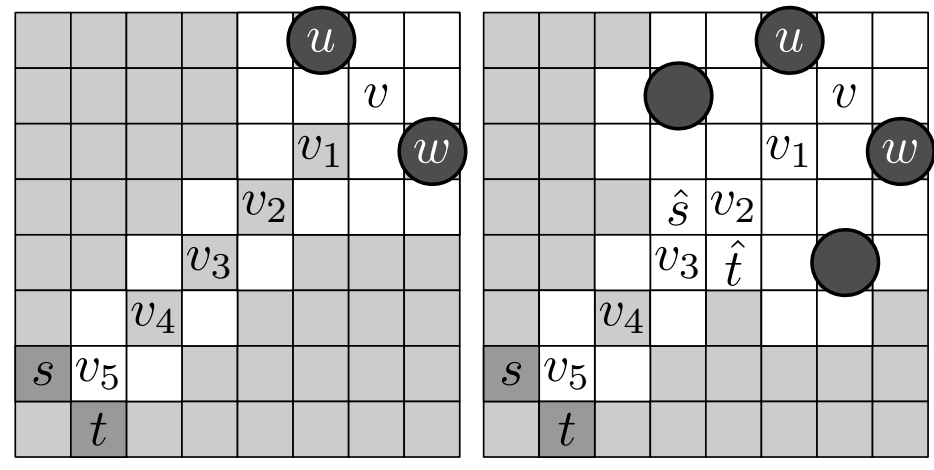

Figure 8: $k=4$ is minimal such that $v_{k+1}=\mathrm{sw}^{k+1}(v)$ is blocked; either $s$ or $t$ belongs to $\tau^{*}$. By minimality of $k$, the elements $\hat{s}$ and $\hat{t}$ are outside $\tau^{*}$.

Let $k \geq 1$ be minimal such that $(\mathrm{sw})^{k+1}(v)$ is blocked by some element in $\tau^{*}$ or in row $m+1$ outside the board. See the picture on the left in Figure 8 for the former case. Note that $v_{j}:=(\mathrm{sw})^{j}(v)$ does not belong to $\tau^{*}$ for $0 \leq j \leq k-1$, because $v_{j+1}=\operatorname{sw}\left(v_{j}\right)$ is not blocked. However, $\left\langle v_{k}\right\rangle$ may be added to or deleted from $\tau$ without the resulting set ending up outside $\Lambda_{0}$. Namely, $v_{k+1}=\mathrm{sw}\left(v_{k}\right)$ is blocked, which means that there is no harm in adding the element. Moreover, the elements $\hat{s}$ and $\hat{t}$ satisfying $\mathrm{s}^{2} \mathrm{w}(\hat{s})=\mathrm{sw}^{2}(\hat{t})=v_{k}$ do not belong to $\tau^{*}$, which means that there is no harm in deleting the element. See the picture on the right in Figure 8, where we also indicated that $\mathbf{s w}^{2}(u)$ and $\mathbf{s}^{2} \mathbf{w}(w)$ belong to $\tau^{*}$.

We also note that the choices of $u$ and $k$ remain unchanged if $v_{k}$ is added to or deleted from $\tau$. Namely, since $k \geq 1$, the index of $v_{k}$ is too large for $v_{k}$ to be a candidate for a new $u$ when added. Moreover, $v_{k}$ is a candidate for a new $w$ only if $k=1$. However, in this case $(\mathrm{nw})^{2}\left(v_{1}\right)$ is blocked by $\mathbf{s w}^{2}(u)$, which makes it impossible for that element to be a candidate for a new $u$. Similarly, $u$ is easily seen to remain unchanged if $v_{k}$ is removed.

In particular, writing $v(\tau)=v_{k}$, we obtain a perfect matching on $\Lambda_{0}$ by pairing $\tau \backslash\{\langle v(\tau)\rangle\}$ and $\tau \cup\{\langle v(\tau)\rangle\}$ for each $\tau \in \Lambda_{0}$.

\section{$6.2 \quad$ Step 2}

We proceed with the remaining family $\Gamma=\Lambda \backslash \Lambda_{0}$. Note that $\Gamma$ consists of all sets $\tau$ in $\Lambda$ such that if $u$ and $w:=(\mathrm{se})^{2}(u)$ belong to $\tau^{*}$ and $u \sim w$, then either $\mathrm{s}^{2} \mathrm{w}(u) \in \tau^{*}$ or $\mathrm{sw}^{2}(w) \in \tau^{*}$. 

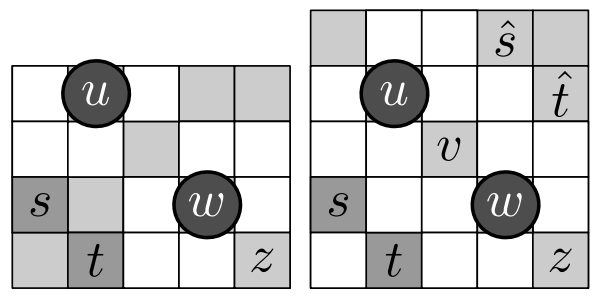

Figure 9: The situation in Step 2. Either $s$ or $t$ belongs to $\tau^{*}$. The element $z$ may or may not belong to $\tau^{*}$, but it is not the case that $z \sim w$.

Let $\Gamma_{0}$ be the subfamily of $\Gamma$ consisting of all sets $\tau$ such that there are two vertices $u$ and $w:=(\mathrm{se})^{2}(u)$ in $\tau^{*}$ such that $u \sim w$ and such that $w \neq z:=\operatorname{se}(w)$, if $z$ is at all in $\tau^{*}$. See the picture on the left in Figure 9 for an illustration. If $z \in \tau^{*}$, then there is no path from $x_{j}$ to $z$ for any $x_{j}$. Namely, assume that there is such a path. Since there is a path from some $x_{i}$ to $w$, and since $x_{i} \nsim x_{j}$, Lemma 5.3 yields that there is a path starting in some $x_{k}(i<k<j)$ that separates the components containing $w$ and $z$. Since there is not room for such a path between $w$ and $z$, we obtain a contradiction.

Write $\Pi=\Gamma \backslash \Gamma_{0}$.

Lemma 6.2 We have that $Z\left(\Gamma_{0}\right)=0$. As a consequence, $Z(\Lambda)=Z(\Gamma)=Z(\Pi)$.

Proof. Among all pairs $(u, w)$ with properties as above, choose the pair such that the row $a$ of $u=:(a, b)$ is maximal and, among the remaining pairs, such that the column $b$ is nonnegative and minimal.

Observe that $\mathrm{e}^{2}(u)=\mathrm{n}^{2}(w)$ does not belong to $\tau^{*}$. Namely, $v:=\operatorname{se}(u)=\operatorname{sw}\left(\mathrm{e}^{2}(u)\right)$ is free in $\tau^{*}$. Moreover, since $\tau$ belongs to $\Gamma$, either $s:=\mathbf{s}^{2} \mathrm{w}(u) \in \tau^{*}$ or $t:=\mathbf{s w}^{2}(w) \in \tau^{*}$. In particular, $\mathrm{sw}(v),(\mathrm{sw})^{2}(v) \notin \tau^{*}$. This means that $\langle v\rangle$ may be added to or deleted from $\tau$ without the resulting set ending up outside $\Gamma_{0}$. Namely, $\operatorname{sw}(v)$ is blocked, which means that there is no harm in adding the element. Moreover, the elements $\hat{s}$ and $\hat{t}$ satisfying $\mathrm{s}^{2} \mathrm{w}(\hat{s})=\mathrm{sw}^{2}(\hat{t})=v$ already have directed edges starting in them and ending in $u$ and $v$, respectively, which means that there is no harm in deleting the element. See the picture on the right in Figure 9 for an illustration. (With some effort, one can prove that either $\hat{s}$ or $\hat{t}$ belongs to $\tau^{*}$, but we do not need this fact for the proof.)

We also note that the choice of $u$ remains unchanged if $v$ is added to or deleted from $\tau$. In particular, writing $v(\tau)=v$, we obtain a perfect matching on $\Gamma_{0}$ by pairing $\tau \backslash\{\langle v(\tau)\rangle\}$ and $\tau \cup\{\langle v(\tau)\rangle\}$ for each $\tau \in \Gamma_{0}$.

\section{Analyzing the family $\Pi$ and settling the proof of Theorem 3.5}

It remains to analyze the family $\Pi$. Our ultimate goal is to prove that (3) is true for all members of $\Pi$. By Lemma 5.4, this will imply that $\Pi$ is empty whenever $n$ is odd and 
hence that $Z\left(Q_{2}\right)=Z(\Pi)=0$.

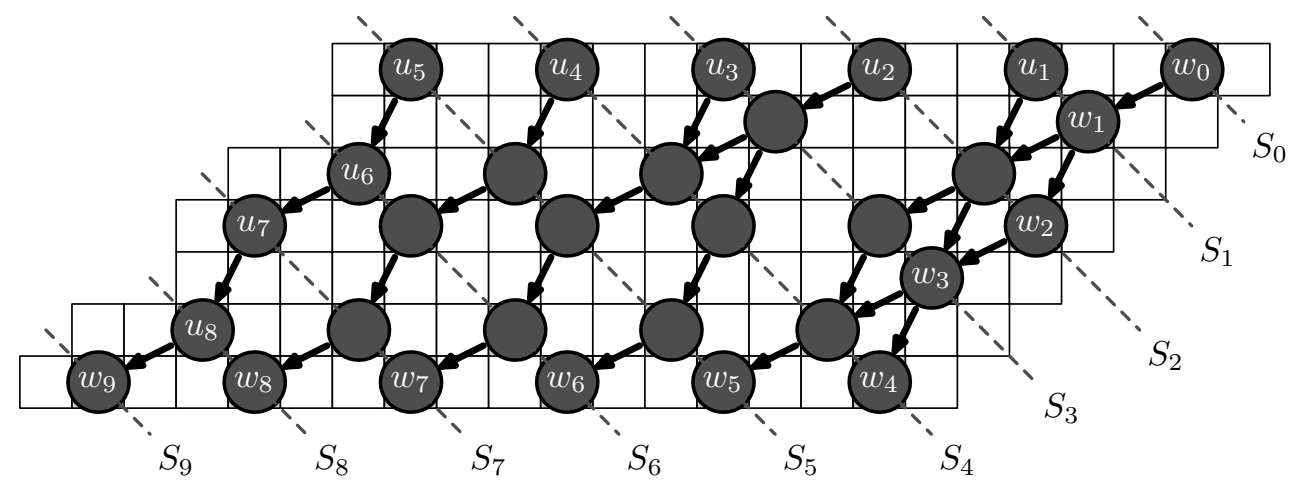

Figure 10: A piece of a set $\tau^{*}$ and its digraph $D\left(\tau^{*}\right)$, where $\tau \in \Pi$. For each $r$, the set $S_{r}$ consists of all disks intersected by the dashed line labeled $S_{r}$. The northwesternmost and southeasternmost elements in $S_{r}$ are $u_{r}$ and $w_{r}$, respectively. The figure illustrates the situation in Section 7.1 for $p=5$ and $q=4$.

Let $\tau \in \Pi$ and consider an equivalence class under $\sim$ in $\tau^{*}$. Let $x_{j}$ be the rightmost element belonging to this class and write $\gamma:=\nu\left(x_{j}\right)$. For each $r \geq 0$, consider the set $S_{r}$ of elements $w \in \tau^{*}$ such that $w \sim x_{j}$ and $\nu(w)=\gamma-3 r$. Note that $S_{r} \backslash\left\{\mathrm{w}^{3 r}\left(x_{j}\right)\right\}$ is the set of elements $w$ such that there is a directed edge from some element in $S_{r-1}$ to $w$. Let the elements $u_{r}$ and $w_{r}$ and the integer $t_{r}$ be such that $w_{r}$ is the southeasternmost element in $S_{r}$ and

$$
u_{r}:=(\mathrm{nw})^{t_{r}}\left(w_{r}\right)
$$

is the northwesternmost element in $S_{r}$. See Figure 10 for an illustration.

Define

$$
c_{i}:= \begin{cases}3 i / 2 & \text { if } i \text { is even; } \\ (3 i-1) / 2 & \text { if } i \text { is odd }\end{cases}
$$

hence $\left(c_{0}, c_{1}, c_{2}, c_{3}, \ldots\right)=(0,1,3,4, \ldots)$.

Lemma 7.1 For each $r$ such that $S_{r}$ is nonempty, let $w_{r}$ be the southeasternmost element in $S_{r}$. Then,

$$
S_{r}=\left\{(\mathrm{nw})^{c_{i}}\left(w_{r}\right): 0 \leq i \leq\left|S_{r}\right|-1\right\}
$$

Proof. For $r=0$, this is evidently true, because $S_{0}=\left\{x_{j}\right\}$. We use induction to prove the statement for all $r$.

Thus suppose that $S_{r}$ has the shape (4), where $r \geq 0$. If $S_{r}$ is empty, then so is $S_{r+1}$. If $S_{r}$ consists of one single element in row $m$, then $S_{r+1}$ is empty, because there are no directed edges starting in such an element. Assume that there is at least one element in $S_{r}$ that does not appear in row $m$. Since this element has an outgoing edge, $S_{r+1}$ is nonempty. We need to prove that $(\mathrm{nw})^{i}\left(w_{r+1}\right) \in S_{r+1}$ if and only if $i \bmod 3 \neq 2$ and $0 \leq i \leq t_{r+1}$. Assume to the contrary that this is not true for some $i$; choose $i$ minimal with this property. Note that $1 \leq i \leq t_{r+1}$. We have three cases. 
- $i \bmod 3=0$ and $p:=(\mathrm{nw})^{i}\left(w_{r+1}\right) \notin S_{r+1}$. We must have that $i<t_{r+1}$, because $(\mathrm{nw})^{t_{r+1}}\left(w_{r+1}\right) \in S_{r+1}$. By minimality of $i, q:=(\mathrm{nw})^{i-1}\left(w_{r+1}\right) \notin S_{r+1}$, which implies that $v:=\mathrm{n}^{2} \mathrm{e}(q)=\mathrm{ne}^{2}(p) \notin S_{r}$. If the northwesternmost element $u_{r}$ of $S_{r}$ is to the northwest of $v$, then $u:=\operatorname{nw}(v) \in S_{r}$ and $w:=\operatorname{se}(v) \in S_{r}$ by induction on $i$, because $w_{r}$ is certainly to the southeast of $v$ and we cannot have two consecutive elements outside $S_{r}$ between $u_{r}$ and $w_{r}$ in $S_{r}$. This means that we have the situation in Figure 7 and hence that $\tau \in \Lambda_{0}$, a contradiction. Hence $u_{r}$ is to the southeast of $v$, meaning that the only possibility for $(\mathrm{nw})^{t_{r+1}}\left(w_{r+1}\right)$ is to appear in the very first row and be equal to $x_{h}$ for some $h<j$. However, this means that $\mathrm{e}^{3}\left(x_{h}\right) \in S_{r}$, which is another contradiction, because this element is certainly to the northwest of $v$.

- $i \bmod 3=1$ and $v:=(\mathrm{nw})^{i}\left(w_{r+1}\right) \notin S_{r+1}$. Again, $i<t_{r+1}$. By minimality of $i$, $w:=(\mathrm{nw})^{i-1}\left(w_{r+1}\right) \in S_{r+1}$ and $z:=(\mathrm{nw})^{i-2}\left(w_{r+1}\right) \notin S_{r+1}$. In particular, $u:=$ $(\mathrm{nw})^{i+1}\left(w_{r+1}\right) \notin S_{r+1}$, because otherwise we would have the situation in Figure 7 or Figure 9 and hence $\tau \in \Lambda_{0}$ or $\tau \in \Gamma_{0}$. Defining $p:=u$ and $q:=v$, we end up with the same situation as in the previous case and obtain a contradiction in exactly the same way.

- $i \bmod 3=2$ and $u:=(\mathrm{nw})^{i}\left(w_{r+1}\right) \in S_{r+1}$. By minimality of $i$, we have that $v:=$ $(\mathrm{nw})^{i-1}\left(w_{r+1}\right) \in S_{r+1}, w:=(\mathrm{nw})^{i-2}\left(w_{r+1}\right) \in S_{r+1}$, and $z:=(\mathrm{nw})^{i-3}\left(w_{r+1}\right) \notin S_{r+1}$. However, this means that we have the situation in Figure 9 and hence that $\tau^{*}$ belongs to $\Gamma_{0}$, a contradiction.

To simplify notation, define $\mathrm{L}(x):=\mathrm{w}^{2} \mathrm{~s}(x)$ and $\mathrm{R}(x)=\mathrm{ws}^{2}(x)$.

Lemma 7.2 For each $r \geq 1$, we have that $\left|S_{r}\right| \geq\left|S_{r-1}\right|-1$, and equality is possible only if either $\left|S_{r-1}\right|$ is even or $w_{r-1}$ appears in the last row $m$.

Proof. The statement is trivial when $S_{r-1}=\emptyset$; hence assume that $S_{r-1}$ is nonempty.

First, assume that $w_{r-1}$ does not appear in row $m$. Then the only way for $S_{r}$ to be smaller than $S_{r-1}$ is that $u_{r}=\mathrm{R}\left(u_{r-1}\right)$ and $w_{r}=\mathrm{L}\left(w_{r}\right)$, meaning that $t_{r}=t_{r-1}-1$. By Lemma 7.1, this implies that we must have that $\left|S_{r}\right|=\left|S_{r-1}\right|-1$ and that $\left|S_{r-1}\right|$ is even. Namely, if $\left|S_{r-1}\right|$ is odd, then $u_{r-1}=(\mathrm{nw})^{3 k / 2}\left(w_{r-1}\right)$ for some integer $k$, which implies that $u_{r}=(\mathrm{nw})^{3 k / 2-1}\left(w_{r}\right)$, a contradiction to Lemma 7.1 .

Next, assume that $w_{r-1}$ does appear in row $m$. The lemma is trivial when $\left|S_{r-1}\right|=1$; hence assume that $\left|S_{r-1}\right| \geq 2$. By Lemma 7.1, this means that $v:=\operatorname{nw}\left(w_{r-1}\right)$ belongs to $S_{r-1}$. Since $\mathbf{s w}(v)$ is blocked and appears in row $m$, we must have that $\mathrm{L}(v)$ belongs to $S_{r}$; note that this is also an element in row $m$. If $\mathrm{L}\left(u_{r-1}\right)$ belongs to $S_{r}$, then $t_{r}=t_{r-1}-1$ and hence $\left|S_{r}\right|=\left|S_{r-1}\right|-1$. Otherwise, $\mathrm{R}\left(u_{r-1}\right)$ belongs to $S_{r}$ and $t_{r}=t_{r-1}-2$, which implies by Lemma 7.1 that $t_{r-1}$ must be a multiple of 3. Namely, otherwise either $t_{r-1}$ or $t_{r}$ would be congruent to 2 modulo 3. Another application of Lemma 7.1 yields that $\left|S_{r}\right|=\left|S_{r-1}\right|-1$. 
Let $p$ be maximal such that $x_{j-p} \sim x_{j}$ and let $q$ be minimal such that $w_{q}$ lies in row $m$. Our goal is to prove (3); hence we restrict to the situation that $p \geq 1$. We divide into two cases depending on whether $p \geq q$ or $q \geq p$.

\subsection{The case $p \geq q$}

Throughout this section, we assume that $p \geq q$.

Lemma 7.3 The sequence $\left(w_{0}, w_{1}, \ldots, w_{q}\right)$ forms a zigzag pattern;

$$
w_{r}= \begin{cases}\mathrm{L}\left(w_{r-1}\right) & \text { if } r \text { is odd; } \\ \mathrm{R}\left(w_{r-1}\right) & \text { if } r \text { is even; }\end{cases}
$$

$1 \leq r \leq q$. Equivalently, $w_{r}$ appears in row $c_{r}+1$ for $0 \leq r \leq q$.

Proof. Assume that $1 \leq r \leq q$ and let $\ell_{r}$ be the row in which $w_{r}$ appears. Since $x_{j-r}$ belongs to $S_{r}$, we have that $t_{r}=\ell_{r}-1$. In particular, $t_{r}=t_{r-1}+1$ if $w_{r}=\mathrm{L}\left(w_{r-1}\right)$, and $t_{r}=t_{r-1}+2$ if $w_{r}=\mathrm{R}\left(w_{r-1}\right)$. By Lemma $7.1, t_{r} \not \equiv 2(\bmod 3) ;$ hence

$$
t_{r}=\left\{\begin{array}{lll}
t_{r-1}+1 & \text { if } t_{r-1} \equiv 0 \quad(\bmod 3) ; \\
t_{r-1}+2 & \text { if } t_{r-1} \equiv 1 \quad(\bmod 3) .
\end{array}\right.
$$

A straightforward induction argument yields the lemma.

Note that Lemma 7.3 implies that $m=c_{q}+1$.

Lemma 7.4 For $q<r \leq p$, we have that $S_{r}=\mathrm{w}^{3}\left(S_{r-1}\right)$ and hence that $S_{r}=\mathrm{w}^{3(r-q)}\left(S_{q}\right)$.

Proof. The element $w_{r}$ must appear in row $m$. Namely, $w_{r-1}$ is in row $m$ by induction on $r$ and Lemma 7.3, and $\mathrm{nw}\left(w_{r-1}\right)$ belongs to $S_{r-1}$ by Lemma 7.1; remember that $x_{j-(r-1)}=(\mathrm{nw})^{m-1}\left(w_{r-1}\right)$ belongs to $S_{r-1}$. Moreover, by assumption, $x_{j-r}$ belongs to $S_{r}$. By Lemma 7.1, the shape of $S_{r}$ is hence identical to that of $S_{r-1}$.

Lemma 7.5 For $p \leq r \leq p+q$, we have that $\left|S_{r}\right| \geq p+q+1-r$ and that $w_{r}$ appears in row $m$. In particular, $S_{p+q}$ is nonempty and contains an element in row $m$.

Proof. By Lemma 7.2 and induction on $r$, it suffices to prove that $w_{r}$ appears in row $m$; by Lemma 7.4 we have that $\left|S_{p}\right|=\left|S_{q}\right|=q+1$.

Assume that $p<r \leq p+q$. By induction, $w_{r-1}$ appears in row $m$ and $\left|S_{r-1}\right| \geq$ $p+q+2-r$. Since the latter is at least two, $v:=\operatorname{nw}\left(w_{r-1}\right)$ belongs to $S_{r-1}$; apply Lemma 7.1. In particular, $\mathrm{L}(v)$ belongs to $S_{r}$, because $\mathrm{R}(v)$ lies in row $m+1$. Since $\mathrm{L}(v)$ lies in row $m$ it must be equal to $w_{r}$, which concludes the proof.

Corollary 7.6 For the situation considered in this section, we have that $d_{j}^{+}-d_{j-p}^{-} \geq 3 p$. In particular, (3) is true for the particular choice of $r$ satisfying $i_{r}=j$.

Proof. Lemma 7.5 yields that $S_{p+q}$ is nonempty and that $w_{p+q}$ belongs to row $m$. Therefore, $w_{p+q}=w^{3 p}\left(w_{q}\right)$, which implies the corollary. 


\subsection{The case $q \geq p$}

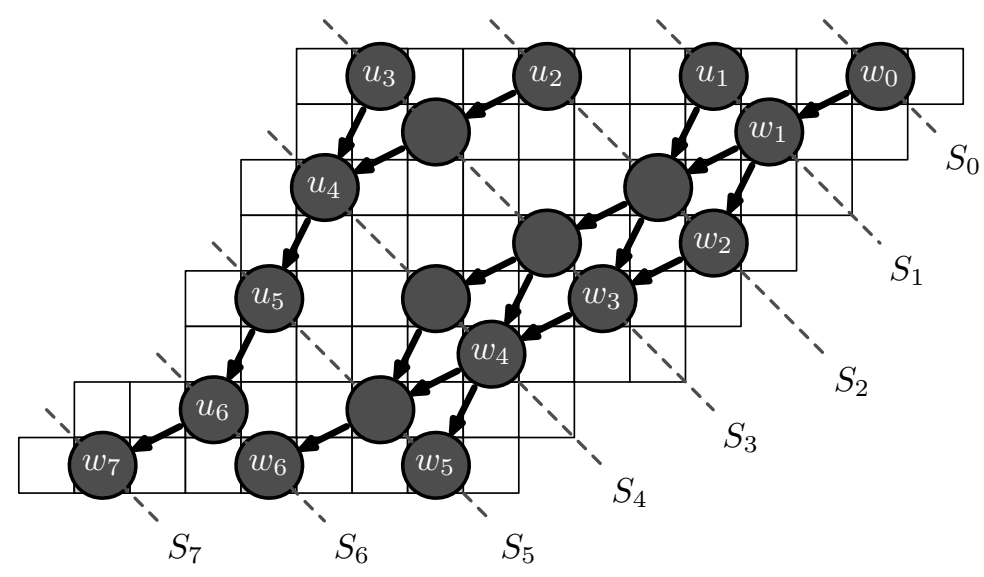

Figure 11: A piece of a set $\tau^{*}$ such that $\tau \in \Pi$ (notation as in Figure 10). The figure illustrates the situation in Section 7.2 for $p=3$ and $q=5$.

Throughout this section, we assume that $q \geq p$. See Figure 11 for an example.

Lemma 7.7 The sequence $\left(w_{0}, w_{1}, \ldots, w_{p}\right)$ forms a zigzag pattern;

$$
w_{r}= \begin{cases}\mathrm{L}\left(w_{r-1}\right) & \text { if } r \text { is odd } \\ \mathrm{R}\left(w_{r-1}\right) & \text { if } r \text { is even }\end{cases}
$$

$1 \leq r \leq q$

Proof. The proof is identical to that of Lemma 7.3.

Lemma 7.8 For $p<r \leq q$, we have that

$$
\begin{cases}\left|S_{r}\right| \geq\left|S_{r-1}\right| & \text { if }\left|S_{r-1}\right| \text { is odd } \\ \left|S_{r}\right| \geq\left|S_{r-1}\right|-1 & \text { if }\left|S_{r-1}\right| \text { is even }\end{cases}
$$

In particular,

$$
\begin{cases}\left|S_{q}\right| \geq p+1 & \text { if } p \text { is even } \\ \left|S_{q}\right| \geq p & \text { if } p \text { is odd }\end{cases}
$$

Proof. This is an immediate consequence of Lemma 7.2 and the fact that $w_{r-1}$ is above row $m$ if $r \leq q$.

Lemma 7.9 For $q \leq r \leq p+q$, we have that $\left|S_{r}\right| \geq\left|S_{q}\right|+q-r$, and $w_{r}$ appears in row $m$. In particular, $S_{p+q-\epsilon}$ is nonempty and contains an element in row $m$, where $\epsilon=0$ if $p$ is even and $\epsilon=1$ if $p$ is odd. 
Proof. The proof is identical to that of Lemma 7.5; for the final statement, apply Lemma 7.8.

Corollary 7.10 For the situation considered in this section, we have that $d_{j}^{+}-d_{j-p}^{-} \geq$ $3(p-\epsilon)$, where $\epsilon=1$ if $p$ is odd and $\epsilon=0$ if $p$ is even. In particular, (3) is true for the particular choice of $r$ satisfying $i_{r}=j$.

Proof. Lemma 7.9 yields that $S_{p+q-\epsilon}$ is nonempty and that $w_{p+q-\epsilon}$ belongs to row $m$. Therefore, $w_{p+q-\epsilon}=\mathrm{w}^{3(p-\epsilon)}\left(w_{q}\right)$, which implies the corollary.

\subsection{Conclusion}

Combining Corollaries 7.6 and 7.10, we obtain that $Z(\Pi)=0$ and hence that $Z\left(Q_{2}\right)=0$. In particular, Theorem 3.5 is proved.

\section{Appendix: Proof of Proposition 3.3}

Proof. (a) To prove that $Z\left(P_{1}\right)=0$, we show that $Z\left(X_{\sigma}\right)=0$ whenever $\sigma \in P_{1}$. By Lemma 3.1, $\hat{\pi}_{e}(\rho)=\hat{\pi}_{e}(\sigma)$ for every $\rho \in X_{\sigma}$. Let $x$ be any element in $\hat{\pi}_{e}(\sigma)$. Another application of Lemma 3.1 yields that $\rho \backslash\{x\}$ belongs to $X_{\sigma}$ if and only if $\rho \cup\{x\}$ belongs to $X_{\sigma}$. In particular, we obtain a perfect matching on $X_{\sigma}$ by pairing $\rho \backslash\{x\}$ and $\rho \cup\{x\}$ for every $\rho \in X_{\sigma}$. It follows that $Z\left(X_{\sigma}\right)=0$.

(b) Our second goal is to compute $Z\left(P_{2}\right)$. If $n$ is odd, then $\pi_{o}(\sigma)$ is empty if and only if the entire first row is empty. As a consequence, $Z\left(P_{2}\right)=Z\left(\Sigma_{m-1, n}\right)$. Suppose $n$ is even. Let $P_{2}^{e}$ be the family of sets $\sigma$ such that all elements in the first row appear in columns with even index. Analogously, let $P_{2}^{o}$ consist of those $\sigma$ such that all elements in the first row appear in columns with odd index. Note that

$$
Z\left(P_{2}\right)=Z\left(P_{2}^{e}\right)+Z\left(P_{2}^{o}\right)-Z\left(\Sigma_{m-1, n}\right)=2 Z\left(P_{2}^{e}\right)-Z\left(\Sigma_{m-1, n}\right)
$$

Here we use the fact that $P_{2}^{e} \cap P_{2}^{o}$ consists of those sets in which the first row is empty and also the fact that $Z\left(P_{2}^{e}\right)=Z\left(P_{2}^{o}\right)$. We conclude that it suffices to prove that $Z\left(P_{2}^{e}\right)$ equals $(-1)^{m n / 4}$ if $m$ is even and 0 otherwise.

For $\sigma \in P_{2}^{e}$, let $j=j_{\sigma} \geq 0$ be minimal such that $j$ is even and $\langle 1, j\rangle$ is free in $\sigma$. If no such $j$ exists, we define $j_{\sigma}=\infty$. Write $x_{\sigma}=\left\langle 1, j_{\sigma}\right\rangle$. It is clear that $x_{\sigma \backslash\left\{x_{\sigma}\right\}}=x_{\sigma \cup\left\{x_{\sigma}\right\}}$ whenever $j_{\sigma}<\infty$. In particular, we may define a matching on $P_{2}^{e}$ by pairing $\sigma \backslash\left\{x_{\sigma}\right\}$ and $\sigma \cup\left\{x_{\sigma}\right\}$ whenever $j_{\sigma}<\infty$.

A set $\sigma$ in $P_{2}^{e}$ is unmatched if and only if $\langle 2, j\rangle$ belongs to $\sigma$ for every even $j$. This implies that there are no elements in columns with odd index in the second row and no elements in columns with even index in the third row. For other positions in the third row and below, there are no restrictions imposed by the presence of the vertices $\langle 2, j\rangle$. 
Writing $P_{2}^{e}(m)=P_{2}^{e}$ and $P_{2}^{o}(m)=P_{2}^{o}$ to indicate the height of the underlying cylinder, we conclude that

$$
Z\left(P_{2}^{e}(m)\right)=(-1)^{n / 2} Z\left(P_{2}^{o}(m-2)\right)=(-1)^{n / 2} Z\left(P_{2}^{e}(m-2)\right) .
$$

Clearly, $Z\left(P_{2}^{e}(0)\right)=1$, and it is an easy task to show that $Z\left(P_{2}^{e}(1)\right)=0$. As a consequence, a simple induction argument yields the desired result.

(c) It remains to compute $Z\left(Q_{1}\right)$. By construction, $Q_{1}$ is empty unless $n$ is a multiple of 3 ; hence assume that $n$ is indeed a multiple of 3 . An independent set $\sigma$ belongs to $Q_{1}$ if and only if $\sigma$ has an element in every third position in the first row. For $i=0,1,2$, let $Q_{1}^{i}$ be the subfamily of $Q_{1}$ consisting of those $\sigma$ that contain $\langle 1, i+3 k\rangle$ for all integers $k$. It is clear that $Z\left(Q_{1}^{0}\right)=Z\left(Q_{1}^{1}\right)=Z\left(Q_{1}^{2}\right)$ and hence that $Z\left(Q_{1}\right)=3 Z\left(Q_{1}^{0}\right)$. From now on, we focus on $Q_{1}^{0}$.

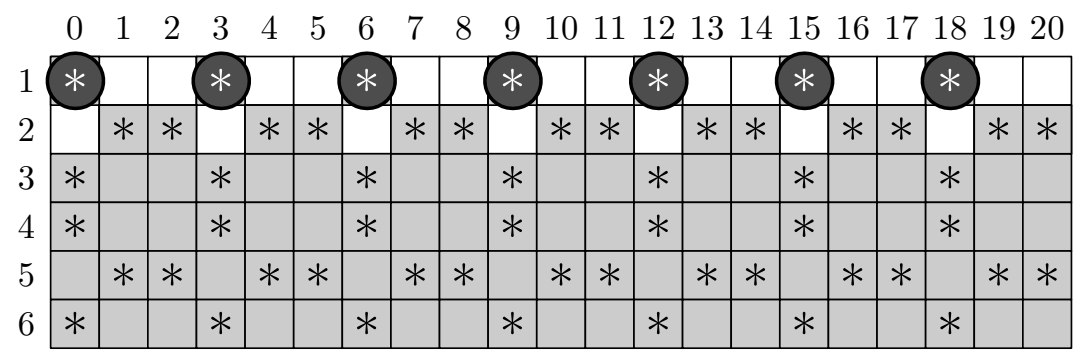

Figure 12: The set $A$ in the proof of (c) consists of all vertices marked with a star, including the present vertices in the first row. Note that the induced subgraph on $A$ is a disjoint union of copies of $K_{2}$ and $K_{1}$.

Divide the vertex set of the underlying graph $C_{m, n}$ into two sets $A$ and $B$. $A$ consists of all vertices $\langle i, j\rangle$ such that either of the following is true.

- $i \bmod 3 \in\{0,1\}$ and $j \bmod 3=0$.

- $i \bmod 3=2$ and $j \bmod 3=\{1,2\}$.

See Figure 12 for an illustration. For any subset $\rho$ of $B$, let $Q_{1}(\rho)$ be the subfamily of $Q_{1}$ consisting of all $\sigma$ such that $\sigma \cap B=\rho$.

First, note that $Q_{1}(\emptyset)$ is the family of independent sets $\sigma$ in the induced graph $C_{m, n}(A)$ such that all elements from $A$ in the first row belong to $\sigma$. We identify three cases, depending on the value of $m \bmod 3$.

- $m \bmod 3=0$. Then $C_{m, n}(A)$ contains isolated vertices in the very last row, which implies that $Z\left(Q_{1}(\emptyset)\right)=0$. This is the situation illustrated in Figure 12.

- $m \bmod 3=1$. Then $C_{m, n}(A)$ is the disjoint union of $n / 3$ copies of $K_{1}$, all appearing in the first row, and $(2 m-2) / 3 \cdot n / 3$ copies of $K_{2}$. It follows that $Z\left(Q_{1}(\emptyset)\right)=$ $(-1)^{n / 3+(2 m-2) n / 9}=(-1)^{n / 3}$. 
- $m \bmod 3=2$. Then $C_{m, n}(A)$ is the disjoint union of $n / 3$ copies of $K_{1}$, all appearing in the first row, and $(2 m-1) / 3 \cdot n / 3$ copies of $K_{2}$. It follows that $Z\left(Q_{1}(\emptyset)\right)=$ $(-1)^{n / 3+(2 m-1) n / 9}=1$.

It remains to prove that $Q_{1}(\rho)=0$ for every nonempty $\rho$. To obtain this, it suffices to find an element $y$ from $A$ strictly below the first row such that no neighbor of $y$ belongs to any set in $Q_{1}(\rho)$. Namely, we may then form a perfect matching on $Q_{1}(\rho)$ by pairing $\sigma \backslash\{y\}$ and $\sigma \cup\{y\}$.

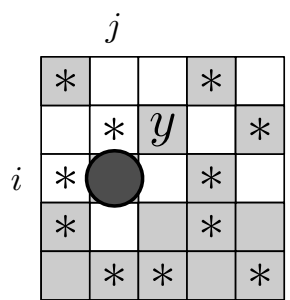

$i \equiv 0, j \equiv 1$

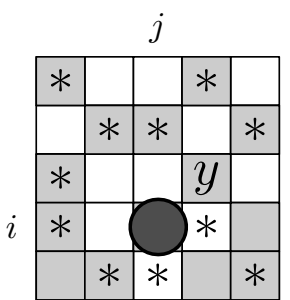

$i \equiv 1, j \equiv 2$

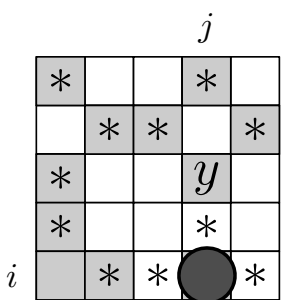

$i \equiv 2, j \equiv 0$

Figure 13: The situation around the vertex $\langle i, j\rangle$ in the proof of (c); stars denote members of $A$. For each case, we indicate the congruence classes modulo 3 of $i$ and $j$. The element $y$ is free in every set in $Q_{1}(\rho)$.

Let $i$ be minimal such that $\rho$ contains some element in row $i$. Pick any element $j$ such that $x:=\langle i, j\rangle \in \rho$. We identify three cases, depending on the value of $i \bmod 3$.

- $i \bmod 3=0$. In this case, $j$ is of the form $3 k+1$ or $3 k+2$ for some integer $k$. If $j=3 k+1$, then no neighbor of the vertex $y:=$ ne $(x)=\langle i-1, j+1\rangle$ belongs to any $\sigma$ in $Q_{1}(\rho)$. Namely, $\mathrm{n}(y)$ and $\mathrm{e}(y)$ both belong to $B$ and are not part of $\rho$ by minimality of $i$. See the picture on the left in Figure 13 for an illustration. The case $j=3 k+2$ is analogous.

- $i \bmod 3=1$. Again, $j$ is of the form $3 k+1$ or $3 k+2$ for some integer $k$. If $j=3 k+2$, then all neighbors of the vertex $y:=$ ne $(x)=\langle i-1, j+1\rangle$ are outside every $\sigma$ in $Q_{1}(\rho)$ by minimality of $i$. See the picture in the middle in Figure 13 for an illustration. The case $j=3 k+1$ is analogous.

- $i \bmod 3=2$. This time, $j$ is of the form $3 k$ for some integer $k$. Since all elements $\langle 2,3 k\rangle$ are blocked, we have that $i \geq 5$. Arguing as before, we deduce that all neighbors of the element $y:=\mathrm{n}^{2}(x)$ are outside every $\sigma$ in $Q_{1}(\rho)$. See the picture on the right in Figure 13 for an illustration. 


\section{References}

[1] R. J. Baxter, Hard squares, Preprint, 2007. arXiv:0709.4324v2

[2] M. Bousquet-Mélou, S. Linusson and E. Nevo, On the independence complex of square grids, J. Algebraic Combin., 27 (2008), no. 4, 423-450.

[3] H. van Eerten, Extensive ground state entropy in supersymmetric lattice models, $J$. Math. Phys. 46 (2005), 123302.

[4] A. Engström, Upper bounds on the Witten index for supersymmetric lattice models by discrete Morse theory, European J. Combin., In Press.

[5] P. Fendley and K. Schoutens, Exact results for strongly-correlated fermions in $2+1$ dimensions, Phys. Rev. Lett. 95 (2005), 046403.

[6] P. Fendley, K. Schoutens, and H. van Eerten, Hard squares with negative activity, J. Phys. A: Math. Gen. 38 (2005), no. 2, 315-322.

[7] L. Huijse and K. Schoutens, Superfrustration of charge degrees of freedom, Proceedings of the XXIII IUPAP International Conference on Statistical Physics, Genova, Italy, 2007. arXiv:0709.4120v1

[8] J. Jonsson, Hard squares with negative activity and rhombus tilings of the plane, Electronic J. Combin. 13 (2006), no. 1, \#R67.

[9] J. Jonsson, Hard squares on grids with diagonal boundary conditions, Preprint, 2008.

[10] D. M. Kozlov, Complexes of directed trees, J. Combin. Theory, Ser. A, 88 (1999), no. $1,112-122$.

[11] J. Thapper, Independence Complexes of Cylinders Constructed from Square and Hexagonal Grid Graphs, Part of Lic Thesis, Linköping University, 2007. arXiv:0812.1165 